\title{
5-Aminosalicylic acid aggravates colitis mimicking exacerbation of ulcerative colitis
}

\author{
Jun Miyoshi ${ }^{1}$, Katsuyoshi Matsuoka ${ }^{1}$, Atsushi Yoshida ${ }^{2}$, Makoto Naganuma $^{1}$, Tadakazu Hisamatsu ${ }^{1}$, \\ Tomoharu Yajima ${ }^{1}$, Nagamu Inoue ${ }^{3}$, Susumu Okamoto ${ }^{1}$, Yasushi Iwao ${ }^{3}$, Haruhiko Ogata ${ }^{4}$, Fumiaki Ueno ${ }^{2}$, \\ Toshifumi Hibi ${ }^{5}$, Takanori Kanai ${ }^{1}$ \\ ${ }^{I}$ Division of Gastroenterology and Hepatology, Department of Internal Medicine, Keio University School of Medicine, Tokyo, ${ }^{2}$ Center for \\ Gastroenterology and Inflammatory Bowel Disease, Ofuna Chuo Hospital, Kamakura, Centers for ${ }^{3}$ Preventive Medicine and ${ }^{4}$ Diagnostic and \\ Therapeutic Endoscopy, Keio University School of Medicine, Tokyo, ${ }^{5}$ Center for Advanced IBD Research and Treatment, Kitasato University \\ Kitasato Institute Hospital, Tokyo, Japan
}

Ulcerative colitis (UC) is one of the major clinical phenotypes of inflammatory bowel diseases. Although 5-aminosalicylic acid (5-ASA) is widely used for UC and its efficacy and safety have been demonstrated, a few patients paradoxically develop a severe exacerbation of colitis by 5-ASA administration. It is crucial to know clinical features including endoscopic findings in this condition for making a correct diagnosis and a prompt decision to withdraw the medication. Here, we report case series with UC exacerbated by 5-ASA. Medical records of 8 UC patients experiencing an exacerbation of colitis after induction of 5-ASA that was improved by the withdrawal of 5-ASA but also re-aggravated by dose increase or re-administration of 5-ASA were reviewed. The patients were newly diagnosed with UC, started 5-ASA and developed an exacerbation in approximately 2 to 3 weeks. They did not appear to have systemic allergic reactions. Seven of the 8 patients had a high fever. Three of 5 patients who undertook total colonoscopy showed right-side-dominant colitis. These findings suggest clinical characteristics in this condition. Further assessment of clinical and endoscopic features in more cases is necessary for establishing diagnostic criteria and understanding underlying mechanisms in those cases where 5-ASA aggravates the colitis. (Intest Res 2018;16:635-640)

Key Words: Colitis, ulcerative; 5-Aminosalicylic acid; Exacerbation; Clinical features; Endoscopic findings

\section{INTRODUCTION}

5-Aminosalicylic acid (5-ASA) is the most widely used antiinflammatory medication for UC. Several types of 5-ASA formulations have been developed to deliver the drug to the colonic mucosa efficiently. In Japan, 3 types of oral 5-ASA formulations are approved for UC; $\mathrm{pH}$-dependent release (Asacol ${ }^{\circledR}$, approved in 2009), time-dependent release (Pentasa $^{\circledR}$, in 1996), and multi-matrix (Lialda ${ }^{\circledR}$, in 2016) formula.

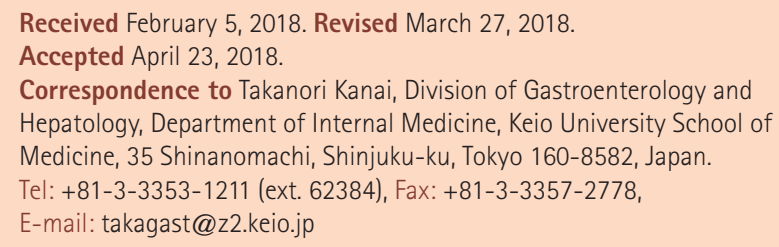

Their clinical efficacy and safety for patients with active UC have been demonstrated in a number of clinical trials. ${ }^{1-3} \mathrm{On}$ the other hand, 5-ASA could paradoxically induce exacerbation of colitis symptoms similar to UC including abdominal pain, diarrhea, and rectal bleeding. ${ }^{4-10}$ Since withdrawing 5-ASA is crucial for this condition, it is essential to suspect this adverse effect of 5-ASA and make a correct diagnosis promptly. For this purpose, it is important to know the clinical features including endoscopic findings in UC patients who develop an exacerbation of colitis symptoms due to 5-ASA, however, clinical features of such patients have not yet been extensively reported. Therefore, we retrospectively reviewed the medical records of patients experiencing UC exacerbations that were thought to be due to 5-ASA administration. Medical charts of UC patients registered in this

\footnotetext{
๑ Copyright 2018. Korean Association for the Study of Intestinal Diseases. All rights reserved.

This is an Open Access article distributed under the terms of the Creative Commons Attribution Non-Commercial License (http://creativecommons.org/licenses/by-nc/4.0)

which permits unrestricted non-commercial use, distribution, and reproduction in any medium, provided the original work is properly cited.
} 
database between 2010 and 2014 were reviewed to identify those who developed aggravation of colitis symptoms by 5-ASA. Our inclusion criteria are: (1) developing symptoms similar to exacerbation of UC including bloody diarrhea and abdominal pain after the introduction of 5-ASA; (2) those symptoms were improved by the withdrawal of 5-ASA; and (3) worsened by dose increase or re-administration of 5-ASA. We assessed details of clinical presentations including endoscopic findings in those subjects.

The study protocol was approved by the ethics committee of the Keio University School of Medicine and conducted in accordance with the Helsinki Declaration of 1975, as revised in 2013.

\section{CASE REPORT}

We identified 8 patients (cases 1-8) who met our inclusion criteria (Table 1). Of the 8 patients, 6 were male, and the median age is 29 years (range, $18-45$ years). All of the 8 patients were newly diagnosed with UC, and 5-ASA was prescribed as the first-line medication (Pentasa ${ }^{\circledR}$ in 3 patients [cases 1-3], Asacol ${ }^{\circledR}$ in 4 patients [cases 5-8], and Asacol ${ }^{\circledR}$ and Pentasa enema ${ }^{\circledR}$ in 1 patient [case 4$]$ ). The median duration of 5-ASA therapy before developing symptoms was 16.5 days (range, 10-22 days). All of them had abdominal pain and bloody diarrhea similar to UC. Seven patients (87.5\%) had a body temperature $\geq 38^{\circ} \mathrm{C}$. The level of CRP was elevated in all the patients (median, $3.17 \mathrm{mg} / \mathrm{dL}$; range, $1.16-18.61 \mathrm{mg} / \mathrm{dL}$ ) when measured at our hospital. Blood examinations did not suggest any 5-ASA-induced systemic adverse events including nephrotoxicity, hepatic dysfunction, or pancreatitis in all the patients.

Five patients (cases $1-3,5$, and 6 ) had been initially hospitalized in other hospitals and treated with prednisolone (PSL) with the diagnosis of exacerbation of UC, and they were referred to our hospital because they did not respond to PSL. One patient (case 4) was referred to our hospital at the diagnosis of severe UC without administration of PSL. The other 2 patients (cases 7 and 8 ) had been treated at the outpatient clinic of our hospital. The 7 hospitalized patients (cases 1-6 and 8) were treated with PSL in addition to withdrawal of 5-ASA. Bacterial infectious colitis was excluded in these 7 hospitalized patients performing fecal bacterial culture and fecal Clostridium difficile toxin test. Immunohistochemistry of mucosal biopsy specimens for cytomegalovirus (CMV) at admission to our hospital was negative in 5 patients (cases 1, 3, 4, 6, and 8) while a positive result in 2 patients (cases 2 and 5). As high-dose PSL therapy had

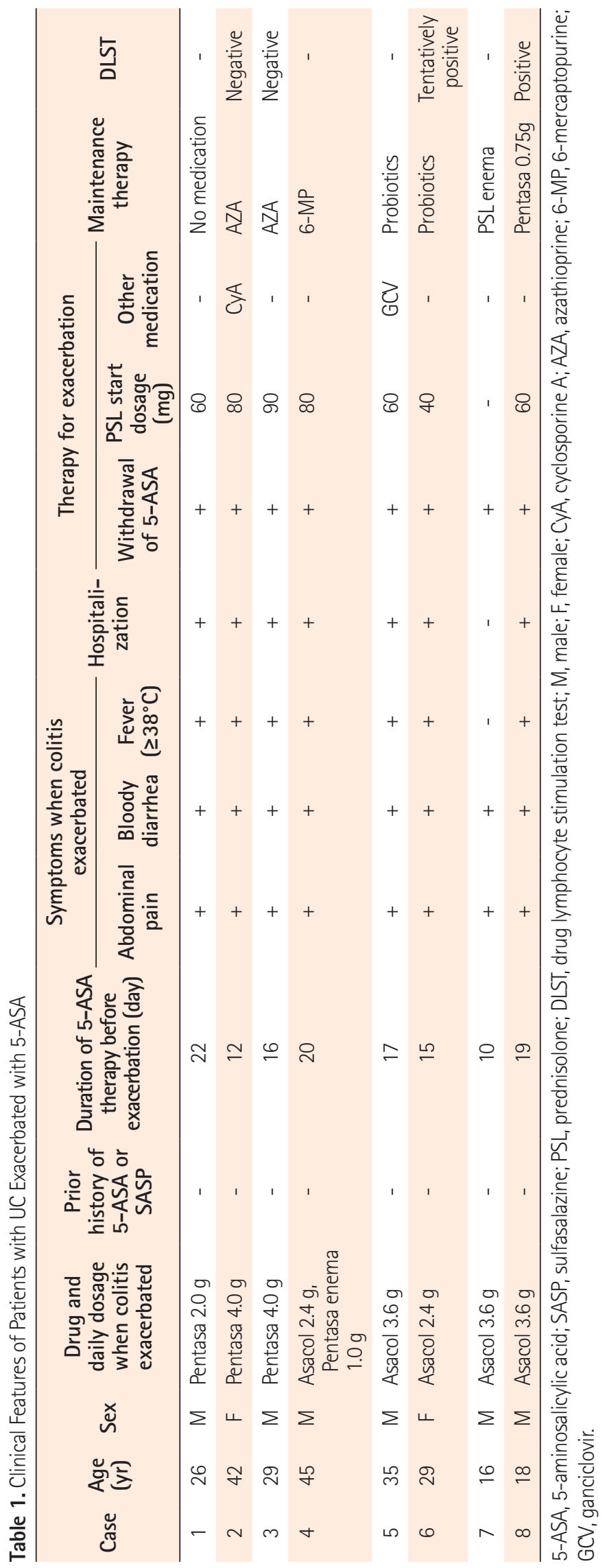


already started in these 2 patients before they were referred to our hospital, we diagnosed them as having reactivation of CMV due to the administration of high-dose PSL and administered ganciclovir in case 5. In these patients, although there is a possibility that CMV reactivation affected their symptoms, the symptoms of both patients were worsened by re-challenge of 5-ASA suggesting that 5-ASA was the primary cause of the exacerbation of colitis rather than CMV. One patient (case 2) received cyclosporine A because acute aggravation and progression to toxic megacolon were concerned. Following the withdrawal of 5-ASA, their symptoms promptly improved and clinical remission was achieved even in patients who had been diagnosed with PSL-resistant severe UC. No patient eventually required surgery. The other patient (case 7), who was afebrile and did not need hospitalization, stopped 5-ASA at the outpatient clinic and improved promptly.

Seven patients (cases 1, 2, and 4-8) were re-administered oral 5-ASA after their colitis symptoms had improved and during PSL was being tapered (cases 1, 2, 4-6, and 8). In case 3 , colitis symptoms were aggravated by increasing dose of 5-ASA in other hospitals and the patient did not take 5-ASA after referred to our hospital from the second hospital. After achieving remission, 3 patients were treated with im- munomodulators (azathioprine in 2 patients [cases 2 and 3], 6-mercaptopurine in 1 patient [case 4]) as maintenance therapy, 3 patients started probiotics (cases 5-7), 1 patient (case 1) received no medication, and 1 patient (case 7) occasionally used PSL enema. The other patient (case 8) developed an exacerbation by re-administration of Asacol ${ }^{\circledR}$ but tolerated Pentasa ${ }^{\circledR}$ with low doses without apparent aggravations. Drug lymphocyte stimulation test (DLST) ${ }^{11}$ was performed in 4 patients (cases 2, 3, 6, and 8). The result was positive in 1 patient (case 8 ), tentatively positive in 1 patient (case 6), and negative in 2 patients (cases 2 and 3).

The 7 hospitalized patients had endoscopic examinations, and total colonoscopy was performed in 5 patients (cases 1 , 2 , and 4-6). There was no evidence for endoscopic findings that suggested CD in any patient. Three out of the 5 patients showed right-side-dominant colitis (cases 2, 4, and 5). One patient, case 4, had left-side-dominant colitis at the initial diagnosis (Fig. 1). However, the colonoscopy performed when referred to our hospital after starting 5-ASA revealed multiple deep ulcers in the ascending colon and cecum (Fig. 2 ). The endoscopic findings of other parts of the colon and the rectum were compatible with moderately active UC and there was no remarkable change in disease activity in these areas.
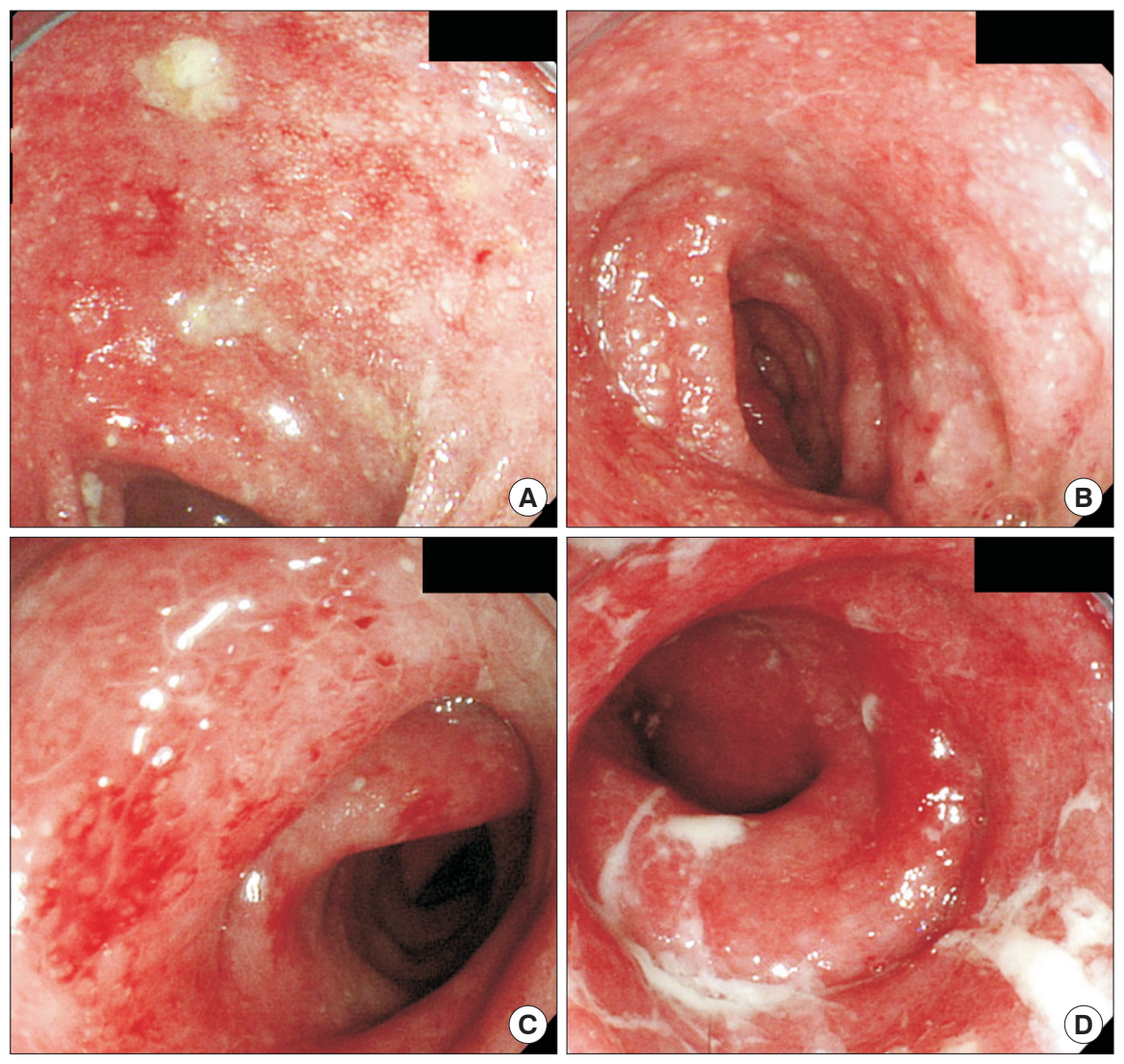

Fig. 1. Endoscopic findings of the case 4 at diagnosis of UC. Total colonoscopy was performed at the previous hospital and the patient was diagnosed with UC. (A) Ascending colon, (B) transverse colon, (C) sigmoid colon, and (D) rectum. 

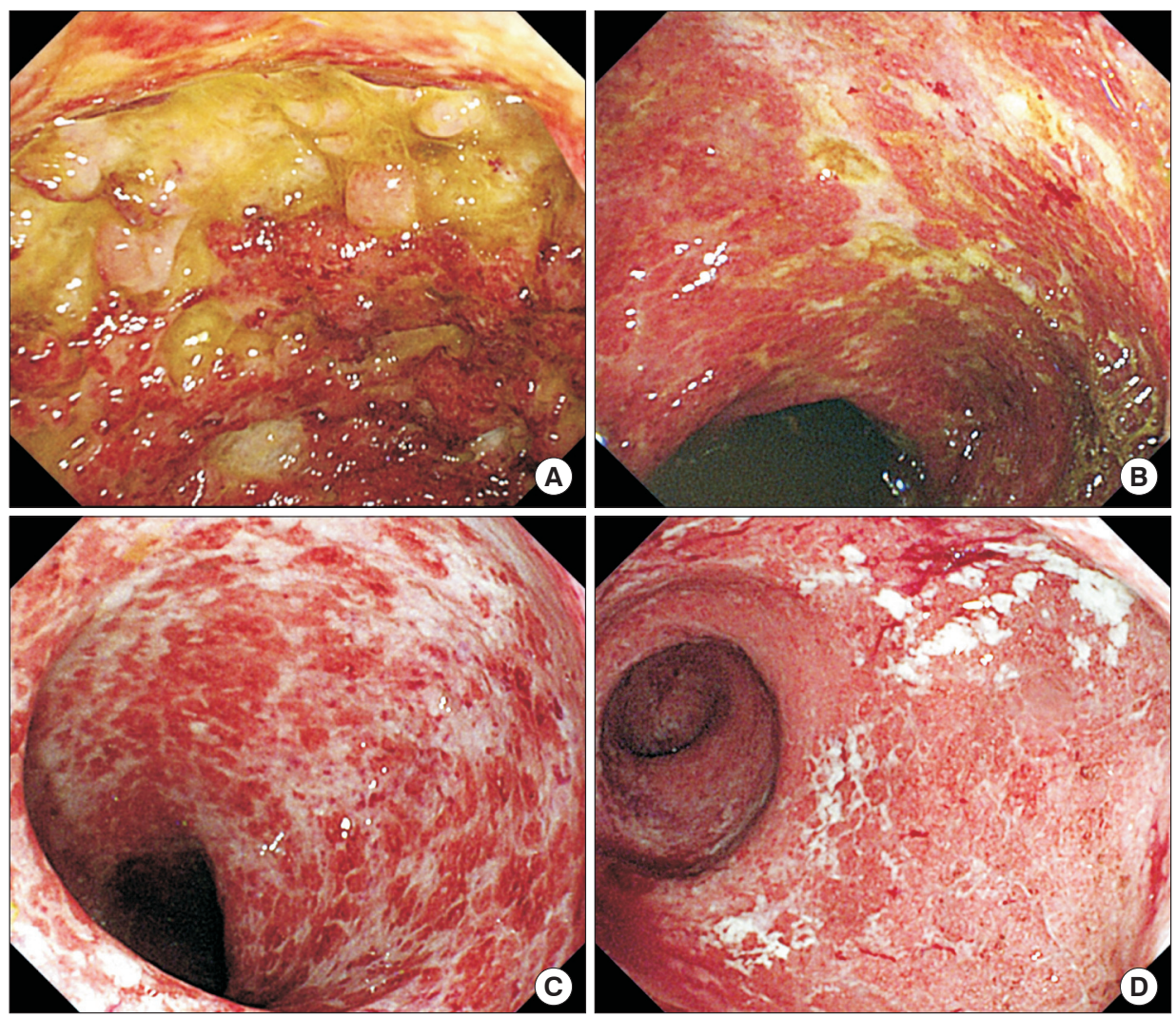

Fig. 2. Endoscopic findings of the case 4 on admission. Total colonoscopy demonstrated the right-side-dominant colitis. (A) Ascending colon, (B) transverse colon, (C) sigmoid colon, and (D) rectum.

\section{DISCUSSION}

5-ASA is a key medication in pharmacological therapy for UC. It has been shown that 5-ASA is effective not only for induction of remission but also for maintenance of remission in patients with UC. ${ }^{12} 5$-ASA is well tolerated and its adverse effects are generally mild including a headache and gastrointestinal symptoms such as nausea, abdominal pain, and watery diarrhea. Meanwhile, some severe adverse events were reported, including arthritis, ${ }_{13}^{13}$ bone marrow suppression, ${ }^{14,15}$ nephrotoxicity, ${ }^{16}$ pancreatitis, ${ }^{17,18}$ pericarditis, ${ }^{13,19}$ pneumonitis, ${ }^{20}$ and exacerbation of colitis symptoms. We found 7 case reports that presented 10 patients with aggravation of bloody diarrhea after 5-ASA administration. ${ }^{4-10}$ Here, we reported 8 UC patients who experienced exacerbation of colitis symptoms related to 5-ASA administration. The strength of our case series is that we included only patients in whom the involvement of 5-ASA in exacerbation of colitis symptoms was confirmed by the re-challenge of 5-ASA. We demonstrated that common clinical features of a 5-ASA-related exacerbation are similar to severe UC including fever, abdominal pain, and bloody diarrhea and the exacerbation develop in approximately 2 to 3 weeks after starting 5-ASA in newly diagnosed patients. It is notable that fever tended to be high $\left(\geq 38^{\circ} \mathrm{C}\right)$ and the level of CRP elevated. These results indicate that an aggravation induced by 5-ASA should be clinically suspected during the initial few weeks of a newly started 5-ASA treatment when symptoms seemed to improve temporally but worsened afterward. If 5-ASA does not improve symptoms, it is difficult to raise a suspicion of 5-ASA-induced aggravation of colitis. It may be necessary to withdraw 5-ASA for a while in such patients to exclude the possibility of 5-ASA-induced aggravation before starting steroids.

Interestingly, we observed right-side-dominant colitis in 3 out of 5 patients who underwent total colonoscopy. Given luminal contents are retained for a long time in the right colon due to peristalsis as well as reversed peristalsis, these endoscopic features suggest the possibility that 5-ASA stay in the right colon for a longer time than in other areas of the colon and the higher concentration of 5-ASA cause more severe mucosal injury with some mechanisms in the right colon. In this case, this pathological condition could be 5-ASAinduced colitis rather than an exacerbation of UC by 5-ASA. While an allergic reaction to 5-ASA has been postulated as a mechanism of worsening by 5-ASA, the common symptoms in our case series, that is, high fever without any skin eruptions or other allergic symptoms, were not typical as a systemic drug allergy. On the other hand, there still be a pos- 
sibility that local allergic reactions occur in colonic mucosa. There are few reports on the endoscopic finding of patients who experienced worsening of colitis symptoms by 5-ASA. Kapur et al. ${ }^{6}$ presented a case of a 30-year-old male patient who was intolerant to Asacol ${ }^{\circledR}$, Pentasa ${ }^{\circledR}$, and Salofalk ${ }^{\circledR}$. The patient, who had been in remission, had a rectal challenge with 5-ASA suppository and developed an exacerbation of symptoms. Colonoscopy and histological examination were performed before and after the challenge, demonstrating that active mucosal inflammation was induced by a single administration of suppository of 5-ASA. Biopsy specimens showed active inflammation with a diffuse infiltrate of neutrophils in the lamina propria. There was no clinical or histological evidence of allergic reaction.

Sturgeon et al. ${ }^{7}$ presented 2 cases of a 34-year-old male patient and a 27 -year-old male patient who were thought to be exacerbated by 5-ASA. In the first case, colonic biopsy specimens showed predominant neutrophil infiltration after 5-ASA treatment. Interestingly, he had shown marked eosinophilic infiltration when he had been intolerant to sulfasalazine (SASP). The second patient initially developed chest pain and myalgias without any change of colitis symptoms with 5-ASA. Later he presented chest pain, myalgias, abdominal pain and frequent bloody stools when the dose of 5-ASA was increased. Then the authors evaluated the mucosal response to local 5-ASA administration and showed that infiltration of the lamina propria with eosinophils and neutrophils was pronounced with worsening edema and crypt destruction. These case reports strongly suggest the possibility that 5-ASA may directly injure colonic mucosa by a mechanism different from an allergic reaction, while the second case reported by Sturgeon et al. ${ }^{7}$ seems a part of the allergic reaction. These findings underscore the importance of determining if the right-side-dominant colitis that we found is characteristic to UC patients aggravated by 5-ASA.

In conclusion, we reported 8 UC patients who experienced exacerbation of colitis symptoms induced by 5-ASA administration and demonstrated their clinical features including endoscopic findings. Our case series have educational implications and contribute to making a correct diagnosis of 5-ASA-related exacerbation. It is important to collect more cases and assess clinical and endoscopic features for establishing diagnostic criteria and understanding underlying mechanisms in this condition.

\section{FINANCIAL SUPPORT}

The authors received no financial support for the research, authorship, and/or publication of this article.

\section{CONFLICT OF INTEREST}

Katsuyoshi Matsuoka received lecture fees from Mitsubishi Tanabe Pharma Co., AbbVie GK, Janssen Pharmaceutical K.K., and Takeda Pharmaceutical Co. Ltd. Tadakazu Hisamatsu received research grants from Mitsubishi Tanabe Pharma Co., EA pharma Co. Ltd., AbbVie GK, JIMRO Co. Ltd., Asahi Kasei Kuraray Medical Co. Ltd., Zeria Pharmaceutical Co. Ltd., Daiichi Sankyo Co. Ltd., Kyorin Pharmaceutical Co. Ltd., Nippon Kayaku Co. Ltd., Astellas Pharma Inc., Takeda Pharmaceutical Co. Ltd., Pfizer Inc., and Mochida Pharmaceutical Co. Ltd., serves as a consultant to EA pharma Co. Ltd., AbbVie GK, Celgene K.K., Janssen Pharmaceutical K.K., Pfizer Inc., Nichi-Iko Pharmaceutical Co. Ltd., and received lecture fees from Mitsubishi Tanabe Pharma Co., Abbvie GK, EA pharma Co. Ltd., Kyorin Pharmaceutical Co. Ltd. and JIMRO Co. Toshifumi Hibi received lecture fees from Mitsubishi Tanabe Pharma Co. Takanori Kanai received research grants from Miyarisan Pharmaceutical Co., Takeda Pharmaceutical Co. Ltd., Mitsubishi Tanabe Pharma Co., Ezaki Glico Co. Ltd., and Otsuka Pharmaceutical Co. Ltd. and lecture fees from Mitsubishi Tanabe Pharma Co., AstraZeneca K.K., Miyarisan Pharmaceutical Co., and Zeria Pharmaceutical Co. Ltd.

\section{AUTHOR CONTRIBUTION}

Conceptualization: JM, KM, TH, TK. Methodology: JM, KM. Formal analysis: JM, KM, AY, MN, TH, TY, NI, SO, YI, HO, FU, TH, TK. Project administration: JM, KM. Visualization: JM, KM. Writing-original draft: JM. Writing-review and editing: KM, AY, MN, TH, TY, NI, SO, YI, HO, FU, TH, TK. Approval of final manuscript: all authors.

\section{REFERENCES}

1. Sninsky CA, Cort DH, Shanahan F, et al. Oral mesalamine (Asacol) for mildly to moderately active ulcerative colitis: a multicenter study. Ann Intern Med 1991;115:350-355.

2. Hanauer S, Schwartz J, Robinson M, et al. Mesalamine capsules for treatment of active ulcerative colitis: results of a controlled trial. Pentasa Study Group. Am J Gastroenterol 1993;88:11881197.

3. Sandborn WJ, Kamm MA, Lichtenstein GR, Lyne A, Butler T, Joseph RE. MMX Multi Matrix System mesalazine for the induction of remission in patients with mild-to-moderate ulcerative colitis: a combined analysis of two randomized, double-blind, placebo-controlled trials. Aliment Pharmacol Ther 2007;26:205215. 
4. Chakraborty TK, Bhatia D, Heading RC, Ford MJ. Salicylate induced exacerbation of ulcerative colitis. Gut 1987;28:613-615.

5. Shanahan F, Targan S. Sulfasalazine and salicylate-induced exacerbation of ulcerative colitis. N Engl J Med 1987;317:455.

6. Kapur KC, Williams GT, Allison MC. Mesalazine induced exacerbation of ulcerative colitis. Gut 1995;37:838-839.

7. Sturgeon JB, Bhatia P, Hermens D, Miner PB Jr. Exacerbation of chronic ulcerative colitis with mesalamine. Gastroenterology 1995;108:1889-1893.

8. Iofel E, Chawla A, Daum F, Markowitz J. Mesalamine intolerance mimics symptoms of active inflammatory bowel disease. J Pediatr Gastroenterol Nutr 2002;34:73-76.

9. Gupta MK, Pollack S, Hutchings JJ. Mesalamine induced symptom exacerbation of ulcerative colitis: case report and brief discussion. World J Gastrointest Pharmacol Ther 2010;1:132-134.

10. Shimodate Y, Takanashi K, Waga E, Fujita T, Katsuki S, Nomura M. Exacerbation of bloody diarrhea as a side effect of mesalamine treatment of active ulcerative colitis. Case Rep Gastroenterol 2011;5:159-165.

11. Pichler WJ, Tilch J. The lymphocyte transformation test in the diagnosis of drug hypersensitivity. Allergy 2004;59:809-820.

12. Ford AC, Achkar JP, Khan KJ, et al. Efficacy of 5-aminosalicylates in ulcerative colitis: systematic review and meta-analysis. Am J Gastroenterol 2011;106:601-616.
13. Lim AG, Hine KR. Fever, vasculitic rash, arthritis, pericarditis, and pericardial effusion after mesalazine. BMJ 1994;308:113.

14. Daneshmend TK. Mesalazine-associated thrombocytopenia. Lancet 1991;337:1297-1298.

15. Nichols TW Jr. Experience with Eudragit-S coated mesalamine (Asacol) in inflammatory bowel disease: adverse events. J Clin Gastroenterol 1994;18:181.

16. Gisbert JP, González-Lama Y, Maté J. 5-Aminosalicylates and renal function in inflammatory bowel disease: a systematic review. Inflamm Bowel Dis 2007;13:629-638.

17. Abdullah AM, Scott RB, Martin SR. Acute pancreatitis secondary to 5-aminosalicylic acid in a child with ulcerative colitis. J Pediatr Gastroenterol Nutr 1993;17:441-444.

18. Fernández J, Sala M, Panés J, Feu F, Navarro S, Terés J. Acute pancreatitis after long-term 5-aminosalicylic acid therapy. Am J Gastroenterol 1997;92:2302-2303.

19. Kaiser GC, Milov DE, Erhart NA, Bailey DJ. Massive pericardial effusion in a child following the administration of mesalamine. J Pediatr Gastroenterol Nutr 1997;25:435-438.

20. Sviri S, Gafanovich I, Kramer MR, Tsvang E, Ben-Chetrit E. Mesalamine-induced hypersensitivity pneumonitis: a case report and review of the literature. J Clin Gastroenterol 1997;24:34-36. 